\title{
Prevalencia de sífilis en mujeres gestantes atendidas por el Programa de Protección a la Gestante (PPG) y reporte de aborto en el Estado de Sergipe, Brasil
}

\author{
Syphilis prevalence in pregnant women served by the \\ Program for Protection of Pregnant Women (PPG) and \\ reporting of abortion in the state of Sergipe, Brazil.
}

Fernando Saab ${ }^{1}$, Carlos Tomaz

Forma de citar: Saab F, Tomaz C. Prevalencia de sífilis en mjeres gestantes atendidas por el Programa de Protección a la Gestante (PPG) y reporte de aborto en el Estado de Sergipe, Brasil. Rev Univ Ind Santander SAlud. 2016; 48(1) 214-220. DOI: http://dx.doi.org/10.18273/revsal.v48n2-2016006 @) (1) $\Theta$

\section{RESUMEN}

Introducción: La sífilis ha representado un importante reto para la salud pública en Brasil, tanto por su alta prevalencia como por las graves secuelas neonatales. Objetivos: Determinar la prevalencia de infección por sífilis y la aparición de abortos involuntarios en mujeres embarazadas, por edad y distribución espacial en el estado de Sergipe en Brasil. Metodología: Estudio transversal descriptivo-retrospectivo, en el que se analizaron 39.807 registros de usuarias del Programa de Protección a la Gestante (PPG) ofrecido en 73 municipios en el estado de Sergipe, en un período aproximado de dos años. Resultados: Fueron seleccionadas 39.807 gestantes, de las cuales 7.538 (18,9\%) reportaron haber abortado, 544 $(1,37 \%)$ fueron halladas con sífilis positiva y $192(0,48 \%)$ presentaron sífilis positiva y reporte de aborto. La proporción aumentó de $25,7 \%$ de mujeres que abortaron y tuvieron cualquier tipo de alteración (HIV, HTLV, Chagas, Hepatitis B, Hepatitis C, Clamidia, Toxoplasmosis, Rubéola, Citomegalovirus, Hipotiroidismo congénito e Fenilcetonuria materna) a 35,3\% en mujeres que abortaron y presentaron confirmación de sífilis positiva, representando un aumento de 1,37 veces de los casos. El Municipio de Carmópolis tuvo el mayor porcentaje de casos con 2,19\%, seguido de Malhador con un $1.30 \%$, Malhada dos Bois con $1.16 \%$, Estância con 1.15\%, Barra dos Coqueiros con $1.14 \%$, Santa Luzia do Itanhy con $1,04 \%$ y Pedrinhas con un $1,00 \%$. Conclusión: Se verificó que en la mayoría de los municipios fue elevado el número de registros de abortos involuntarios en mujeres gestantes. La prevalencia gestacional de sífilis fue superior a la aceptada por la Organización Mundial de la Salud (OMS). Por medio del PPG se verificó que la sífilis continúa siendo un importante problema de salud durante la gestación, y que las mujeres con sífilis presentan una mayor frecuencia de abortos.

Palabras clave: Sífilis, gestantes, prevalencia, aborto.

1. Universidade de Brasília. Brasília, DF, Brasil.

Correspondencia: Laboratório de Neurociência e Comportamento, Departamento de Ciências Fisiológicas, Instituto de Biologia, Universidade de Brasília, Campus Darcy Ribeiro, IB- Bloco G, 1 Andar, Brasília, DF 70910-900, Brasil. E-mail: ctomaz@unb.br 


\section{ABSTRACT}

Introduction: Syphilis has represented a major challenge to public health in Brazil for its prevalence and serious perinatal sequel. Objectives: To know the prevalence of syphilis infection and the occurrence of abortion in pregnant women, by age and spatial distribution in the state of Sergipe. Methodology: This is a cross sectional descriptive study, retrospective in which were analyzed 39.807 entries of users of the Program for the Protection of Pregnant women (PPP) in 73 municipalities of the state of Sergipe in a period of approximately two years. Results: We screened 39.807 pregnant women, these $7.538(18,9 \%)$ said they have aborted, $544(1,37 \%)$ had syphilis positive and $192(0,48 \%)$ pregnant women had syphilis positive and abortion declared. The proportion rose from $25,7 \%$ in the number of women who aborted and had any type of change to $35,3 \%$ in women who aborted and confirmed positive syphilis, an increase of 1,37 in the number of cases. The municipality of Carmópolis had the highest percentage of cases 2,19\%, followed by Malhador 1,30\%, Malhada dos Bois 1,16\%, Estância 1,15\%, Barra dos Coqueiros 1,14\%, Santa Luzia do Itanhy 1,04\% e Pedrinhas 1\%. Conclusion: It was found that was high the registration of abortions in pregnant women in the most part of the municipalities. The rates of prevalence and incidence of syphilis were higher than those accepted by OMS. Through the PPP can be verified that syphilis may be an associated factor to the occurrence of high incidence of abortion and perinatal morbidity and mortality in the state of Sergipe in Brazil.

Keywords: Syphilis, pregnant women, prevalence.

\section{INTRODUCCIÓN}

Considerada como una enfermedad sexualmente transmisible, según Campos $^{1}$, la sífilis ha venido desafiando los servicios de salud pública a lo largo de los años ${ }^{2}$. A pesar de conocerse el agente etiológico, Treponema pallidum ssp. pallidum, su diagnóstico sigue siendo controvertido y casi siempre está basado en criterios poco fundamentados ${ }^{3}$. Para el médico obstetra, la importancia de esta enfermedad trasciende sus aspectos clínicos (lesión primaria, lesiones cutáneas y neurosífilis), aduciendo al complejo abordaje de la transmisión vertical de su agente etiológico ${ }^{4}$. Cuando la mujer adquiere sífilis durante el embarazo, pueden ocurrir eventos como: aborto espontáneo, muerte fetal, prematuridad, fetal hidrópico, recién nacidos sintomáticos y asintomáticos. Cuanto más temprano afecte al feto, más graves son las consecuencias 5 . Las alteraciones patológicas observadas en mujeres embarazadas son las mismas que ocurren en aquellas no embarazadas ${ }^{6}$.

La sífilis en el embarazo constituye un problema importante principalmente por dos razones, una de ellas es la gravedad de las lesiones que puede provocar en el feto cuando no es tratada la infección, y la otra es la posibilidad real de hacer profilaxis medicamentosa de la sífilis congénita durante un control prenatal adecuado ${ }^{6}$.

La sífilis se configura como una enfermedad difícil de entender. La historia natural puede llevar décadas en un individuo y el diagnóstico de laboratorio y la conducta terapéutica son, usualmente definidos serológicamente, y no clínica o bacteriologicamente? Se considera una enfermedad con alto "camuflaje", con cerca de 65 diagnósticos diferenciales reportados. Por otro lado, es una enfermedad en la que el paciente se queja poco, en realidad, mucho menos de lo que se podría esperar de una enfermedad tan grave o potencialmente grave ${ }^{8}$.

En el contexto Brasilero es conocido el alto número de subdiagnóstico y subnotificación de sífilis, la poca visibilidad de su gravedad y la baja calidad de la atención a las mujeres embarazadas, así como una prevalencia de sífilis de alrededor de 1,7\%, una incidencia de sífilis congénita de 1,3 casos/1000 NV, estimándose de 12 a 15 por $1000 \mathrm{NV}$, y una elevada mortalidad por sífilis congénita ${ }^{9,2}$.

En virtud del alto índice de casos de sífilis en el Estado de Sergipe, el bajo nivel socioeconómico y la prevalencia que permanece desde el siglo pasado, con la presente investigación se pretendió proporcionar, a las autoridades de salud, información que contribuirá al desarrollo o mejora de programas de prevención de transmisión de sífilis.

El propósito del presente estudio fue abordar epidemiológicamente la prevalencia de sífilis en mujeres gestantes y la aparición de abortos involuntarios en mujeres embarazadas, por edad y distribución espacial en el estado de Sergipe en Brasil durante un período de 21 meses, contemplando la totalidad de los 73 municipios adheridos y de mujeres registradas en el SGPPG. 


\section{METODOLOGÍA}

Estudio de tipo transversal descriptivo, retrospectivo, en el cual se analizaron 39.807 registros de usuarias del PPG, atendidas en 73 Municipios del Estado de Sergipe, en un período de 21 meses.

Para el presente estudio se contó con la participación de todas las mujeres gestantes inscritas en la primera consulta del control prenatal, independientemente de su edad cronológica y edad gestacional. Sólo fueron analizados los datos de quienes se ajustaban a los criterios de registro correcto y completo según la ficha de recolección de la información. Después del registro de todos los datos y la implementación de los exámenes, fueron identificadas las mujeres embarazadas con sífilis y que habían abortado. El diagnóstico fue realizado mediante la prueba de detección ELISA, donde el material tomado fue sangre seca en papel filtro (SS 903), y confirmado por ELISA, VDRL y FTA-ABS en suero.

Se evaluaron en el presente estudio, los datos generados a partir del Sistema de Gestión del Programa de Protección a la Gestante (SGPPG), creado específicamente para la administración del programa, de todos los municipios adheridos en el período de 21 meses consecutivos (2005 y 2007) desde el inicio de su funcionamiento, así como todas las mujeres embarazadas registradas en el sistema.

El sistema fue desarrollado en ambiente Windows, en el lenguaje Delph, permitiendo exportar datos en patrones compatibles con los estándares Tabwin y Epinfo de fácil manejo. El sistema contó como entrada la Ficha de Registro de la Gestante. Estas informaciones originadas en las unidades básicas de salud, fueron digitadas diariamente, siendo el SGPPG el que generaba los respectivos informes. Participaron de la presente evaluación los municipios adheridos al programa y que además reportaron su información al sistema. Hasta el mes de mayo de 2007 fueron registradas en el sistema únicamente las gestantes identificadas por las unidades básicas de salud (UBS).

Las variables obtenidas y estudiadas fueron: Edad gestacional, rango de edad, número de abortos, número de embarazos, número de mujeres que realizaron el control prenatal y número de casos positivos para sífilis.

Para el análisis de los datos fue creado un banco de información en una planilla de cálculo del programa Microsoft para Windows Excel ${ }^{\circledR}$, con datos ofrecidos por el SGPPG y se usó el programa Statical Analyses Sytem (SAS) ${ }^{\circledR}$ para el análisis de la información.

\section{RESULTADOS}

Durante el periodo de recolección de datos se encontró que del total previsto de registros en el período (55.818) -número total de mujeres embarazadas en el períodofueron analizados 39.807, representando un $71,31 \%$ dentro de los 73 municipios de referencia.

Para noviembre de 2006, se registró una caída en la cobertura, debido a los cambios generados en la planta de servidores en todas las unidades de salud, siendo sustituidos servidores contratados por servidores concursados.

De las 39.807 gestantes analizadas, $637(1,60 \%)$ eran de 14 años o menos, $8.882(22,31 \%)$ de 15 a 19 años, $20.760(52,15 \%)$ de 20 a 29 años, $7.889(19,82 \%)$ de 30 a 39 años, 950 (2,39\%) entre los 40 y 49 años, 33 $(0,08 \%)$ mujeres mayores de 50 años y finalmente 656 $(1,65 \%)$ no informaron su edad. La mayor concentración de gestantes estuvo en el rango de 20 a 29 años, con más de la mitad de las mujeres embarazadas.

En cuanto a la distribución por edad gestacional, se halló que: $11.167(28,05 \%)$ mujeres estaban hasta con 12 semanas de embarazo, 16.362 (41,10\%) entre 13 y 24 semanas, $3.011(7,56 \%)$ entre 25 y 36 semanas, y $199(0.50 \%)$ con 37 o más semanas. Se identificó que $9.068(22.78 \%)$ mujeres no informaron o no conocían su edad gestacional.

Del total de 39.807 mujeres embarazadas, 7.538 $(18,94 \%)$ reportaron haber tenido aborto en algún momento de su vida, de las cuales 5.834 (77,39\%) informaron un aborto; $1.185(15,72 \%)$ dos abortos; $329(4,36 \%)$ tres abortos y $190(2,52 \%)$ cuatro o más abortos. La prevalencia calculada de abortos ocurrida en el presente estudio fue de 189,4 por cada 1000 mujeres embarazadas

Del total de 39.807 mujeres embarazadas, en 544 $(1,37 \%)$ se confirmó sífilis en suero, representando una prevalencia de 13,7 casos por cada 1.000 mujeres embarazadas.

Del grupo de mujeres embarazadas con sífilis confirmada en suero (544), $192(35,29 \%)$ presentaron abortos en algún momento de su vida. Cuando se analizó el número de abortos por grupo de edad, no hubo ocurrencia de aborto en ninguna gestante con hasta 14 años, en las mujeres entre los 15 y 19 años se reportaron tres abortos $(7,69 \%)$ de los 39 casos conocidos con sífilis; en el rango de 20 a 29 años fueron 
89 los abortos $(33,84 \%)$ del total de 263 gestantes. De los 30 a los 39 años fueron identificados 77 casos de aborto $(39,89 \%)$ de un total de 193 mujeres. Entre los 40 y 49 años ocurrieron 18 abortos $(45,00 \%)$, de las 40 mujeres con sífilis. Finalmente, por encima de los 50 años no se conoció ocurrencia de aborto. No se contó con información de los cinco casos restantes en cuanto a edad. Los resultados se muestran en la Tabla 1.

Tabla 1. Distribución por rango de edad y cantidad de abortos en mujeres embarazadas con sífilis confirmada.

\begin{tabular}{|c|c|c|c|c|c|c|}
\hline 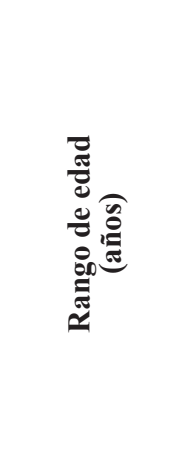 & 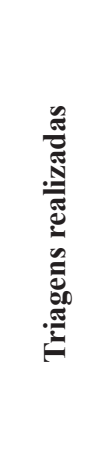 & 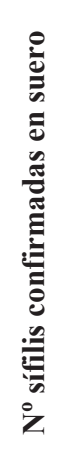 & 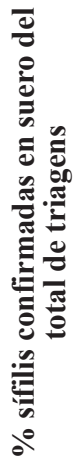 & 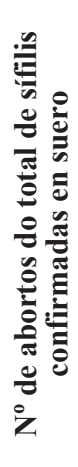 & 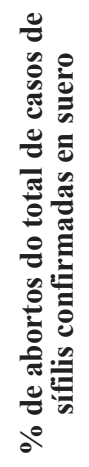 & 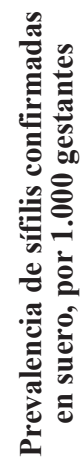 \\
\hline Hasta 14 & 637 & 0 & 0,00 & 0 & 0,00 & 0,00 \\
\hline $15-19$ & 8.882 & 39 & 0,43 & 3 & 7,69 & 4,39 \\
\hline $20-29$ & 20.760 & 263 & 1,26 & 89 & 33,84 & 12,67 \\
\hline $30-39$ & 7.889 & 193 & 2,44 & 77 & 39,89 & 24,46 \\
\hline $40-49$ & 950 & 40 & 4,21 & 18 & 45,00 & 42,11 \\
\hline 50 ó + & 33 & 0 & 0,00 & 0 & 0,00 & 0,00 \\
\hline $\begin{array}{l}\text { No } \\
\text { respondieron }\end{array}$ & 656 & 9 & 1,37 & 5 & 55,55 & 13,72 \\
\hline Total & 39.807 & 544 & 1,37 & 192 & 35,29 & 13,67 \\
\hline
\end{tabular}

Fuente: IMASC/APAE/SE

Cuando fue analizada la cantidad de abortos por edad gestacional en las mujeres con sífilis, hasta las 12 semanas de gestación se encontraron 56 abortos $(39,16 \%)$ del total de 143 mujeres con sífilis; en las mujeres entre las 13 y 24 semanas, se presentaron 94 casos de abortos $(41,96 \%)$ de un total de 224 mujeres; en la edad gestacional entre las 25 y 36 semanas fueron 19 óbitos $(35,84 \%)$ de un total de 53 mujeres. En la edad gestacional superior a las 37 semanas fue reportado 1 óbito $(25,00 \%)$ del total de 4 mujeres embarazadas con sífilis. 22 de los casos de mujeres embarazadas con sífilis no reportaron el dato de edad gestacional, como se detalla en la Tabla 2.
Tabla 2. Distribuición por edad gestacional y cantidad de abortos en mujeres embarazadas con sífilis confirmada.

\begin{tabular}{|c|c|c|c|c|c|c|}
\hline 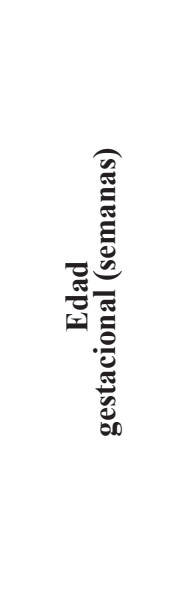 & 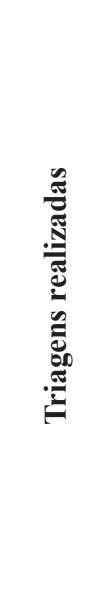 & 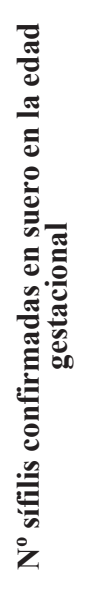 & 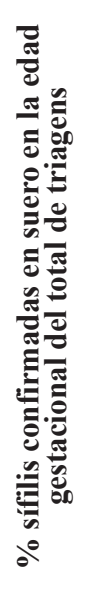 & 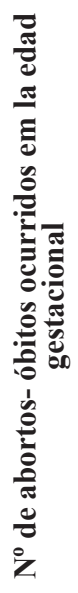 & 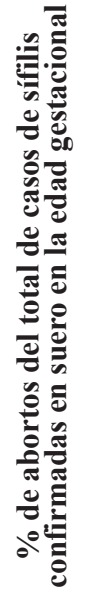 & 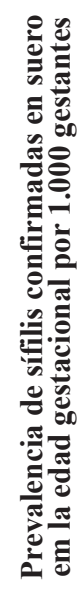 \\
\hline Hasta 12 & .167 & 143 & 1,28 & 56 & 39,16 & 12 \\
\hline $13-24$ & 16.362 & 224 & 1,36 & 94 & 41,96 & 13,69 \\
\hline $25-36$ & 3.011 & 53 & 1,76 & 19 & 35,84 & 17,60 \\
\hline 37 ó + & 199 & 4 & 2,01 & 1 & 25,00 & 20,10 \\
\hline $\begin{array}{c}\text { No } \\
\text { respondieron }\end{array}$ & 9.068 & 120 & 1,32 & 22 & 18,33 & 13,23 \\
\hline Total & 39.807 & 544 & 1,36 & 192 & 35,29 & 3 , \\
\hline
\end{tabular}

Fuente: IMASC/APAE/SE

En cuanto a la distribución geográfica, el municipio de Carmópolis reportó el mayor porcentaje de los casos, $2,19 \%$, considerado muy alto para el estudio, seguido de Malhador con 1,30\%, Malhada dos Bois con 1,16\%, Estância con 1,15\%, Barra dos Coqueiros con 1,14\%, Santa Luzia do Itanhy con $1,04 \%$ y Pedrinhas con 1,00\%. Los municipios, Amparo de São Francisco, Brejo Grande, Canhoba, Carira, Feira Nova, Garuru, General Maynard, Maruim, Moita Bonita, Monte Alegre de Sergipe, Pacatuba, Pedra Mole, Pirambu, Porto da Folha, Riachuelo, Santana do São Francisco, Santa Rosa de Lima, Santo Amaro das Brotas, São Francisco, São Miguel do Aleixo y Telha no reportaron casos de mujeres embarazadas con sífilis positiva y al menos 1 aborto.

En número de casos por municipio, Estância registró la mayor cantidad, tanto en casos positivos de sífilis sin aborto (52), como en casos positivos de sífilis en mujeres gestantes con reporte de aborto (22).

Por otra parte, del total de las 39.807 gestantes, 2.400 $(6,02 \%)$ presentaron por lo menos una patología confirmada en suero, revelando una prevalencia de 60,29 casos por cada 1.000 mujeres gestantes. 
De las 2.400 mujeres embarazadas con cualquier patología confirmada en suero (HIV, HTLV, Chagas, Hepatitis B, Hepatitis C, Clamidia, Toxoplasmosis, Rubéola, Citomegalovirus, Hipotiroidismo congénito e Fenilcetonuria materna), en 617 (25,70\%) se presentaron abortos. Al analizar el número de abortos por rango de edad, se encontró 1 caso $(5,88 \%)$ del total de 17 mujeres embarazadas en el rango de hasta los 14 años, para el rango de los 15 a los 19 años ocurrieron 37 abortos $(9,36 \%)$ del total de 395 mujeres embarazadas, entre los 20 y 29 años fueron 311 casos de aborto (24,74\%) del total de 1.357 mujeres. En el rango de edad de los 30 a 39 años fueron $218(37,07 \%)$ del total de 588. Entre los $40 \mathrm{y}$ los 49 años fueron 38 (41.22\%) del total de 90 mujeres. Por encima de 50 años no se reportó evento de aborto. 12 mujeres embarazadas no reportaron su edad y no fue posible establecer su rango de edad, de acuerdo con lo señalado en la Tabla 3.

Tabla 3. Distribución por rango de edad y cantidad de abortos por patologia confirmada.

\begin{tabular}{|c|c|c|c|c|c|c|}
\hline 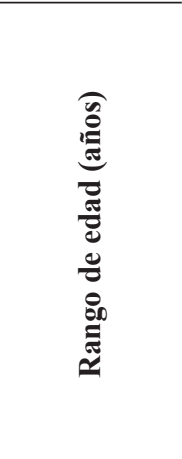 & 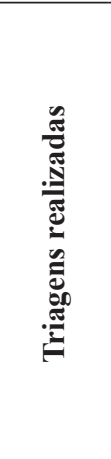 & 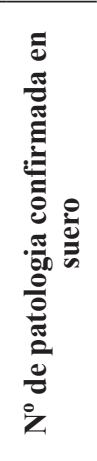 & 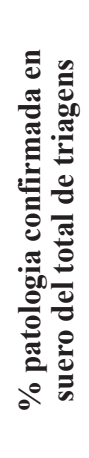 & 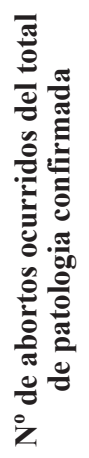 & 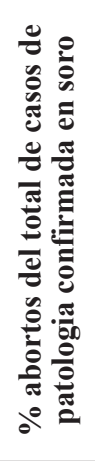 & 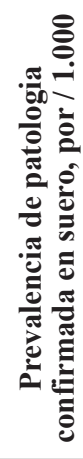 \\
\hline Has & 57 & 7 & 2,66 & & 88 & 26 \\
\hline 10 & 8.382 & 395 & 4,71 & 37 & 9,36 & 44 \\
\hline $20-29$ & 20.760 & 1.257 & 6,05 & 311 & 24,74 & 60,55 \\
\hline $30-39$ & 889 & 588 & 7,45 & 218 & 37,07 & 12 \\
\hline $40-49$ & 950 & 90 & 9,47 & 38 & 41,22 & 94,74 \\
\hline 50 ó + & 33 & 0 & 0,00 & 0 & 0,00 & 0,0 \\
\hline $\begin{array}{c}\text { No } \\
\text { spondieron }\end{array}$ & 656 & 53 & 8,07 & 12 & 22,64 & 80,7 \\
\hline Total & 39.807 & 2.400 & 6,02 & 617 & 25,70 & 00 \\
\hline
\end{tabular}

Fuente: IMASC/APAE/SE

\section{DISCUSIÓN}

Las infecciones congénitas y perinatales representan un problema de relevancia, ya que son prevenibles y sus causas principales refieren la ausencia o negligencia de acciones en cuanto a un diagnóstico precoz y tratamiento correcto y temprano de la enfermedad materna. Por tanto, el conocimiento de los datos de prevalencia durante la gestación, el parto y el periodo neonatal son de igual manera tan esenciales como las acciones preventivas, educativas y profilácticas al comprometimiento fetal. Cuanto más tarde sea el diagnóstico y tratamiento de la infección materna, mayor serán las dificultades de concluir el tratamiento oportunamente, con el fin de que ocurra la prevención de la transmisión vertical en el recién nacido ${ }^{10}$.

En Brasil, de acuerdo con el Proyecto Centinela Parturiente (Projeto Sentinela Parturiente) ${ }^{11}$ la sífilis congénita se muestra como un evento de salud de gran magnitud, presentando indicadores desfavorables en términos de su control, convirtiendo a su abordaje en una prioridad de política pública. En 1986, la sífilis congénita se convirtió en notificación obligatoria en Brasil ${ }^{12}$. Aunque existe subnotificación, entre los años 1998 y 2006 fueron notificados cerca de 36.000 casos de la enfermedad en población general. Con el aumento progresivo de la notificación, la incidencia pasó de 1,3 casos por cada 1.000 nacidos vivos, en el año 2000 , a 1,7 casos por cada 1.000 nacidos vivos en el 2005. Entre los casos reportados en el 2005, el $79,8 \%$ de las madres tuvieron atención prenatal. De este grupo, el 53,8\% fueron casos diagnosticados con sífilis durante el embarazo y en sólo el 13,2\% las parejas fueron tratadas ${ }^{13}$. En el mismo 2005 se observó una tasa de mortalidad de 2 óbitos por cada 100.000 menores de 1 año, demostrando este dato un insuficiente control de la gravedad de la enfermedad en todo el territorio nacional ${ }^{13}$.

Los resultados apuntan a que el $35,3 \%$ de las mujeres gestantes con diagnóstico positivo de sífilis ya habían abortado. De acuerdo con la literatura, ${ }^{14,15}$ hasta el $90 \%$ de los fetos afectados por la enfermedad son asintomáticos al nacer. La intensidad de las manifestaciones clínicas de la sífilis en los recién nacidos depende de la treponemia materna durante el periodo de embarazo en el que se produjo la infección fetal. Por lo tanto, en la etapa reciente (fase primaria o secundaria de la enfermedad) la transmisión perinatal va de un $70 \%$ a un $100 \%$, reduciéndose para un $40 \%$ a $80 \%$ a principios de la fase latente y de un $10 \%$ a un $30 \%$ en las fases latente tardía y terciária ${ }^{14,16}$. Los niños no tratados durante el período neonatal a menudo tienden a enfermarse en los primeros meses de vida y pueden desarrollar cuadros graves de orden clínico $^{17,18}$. En particular, la sífilis congénita se ha asociado con mayores tasas de prematuridad y bajo peso al nacer ${ }^{14,15,17}$.

El acompañamiento prenatal fue reportado por casi el $100 \%$ de las mujeres estudiadas; sin embargo, el número de casos sigue siendo extremadamente elevado. 
Dicho fenómeno posiblemente esté relacionado con una captación excesivamente tardía de los casos de mujeres embarazadas estudiados en los servicios prenatales, ya que sólo el $28,1 \%$ de ellos informó de la primera visita prenatal antes de la $12^{\mathrm{a}}$ semana de embarazo. Esta situación, además de contribuir a un diagnóstico tardío de la infección materna, llega a dificultar la implementación de un tratamiento a tiempo que pueda prevenir la transmisión materno-fetal del treponema ${ }^{10}$.

Trabajos en la literatura reportan que los problemas y deficiencias en la atención prenatal son características comunes entre los países en desarrollo y directamente influenciadas por el desarrollo económico, cultural y tecnológico de la región ${ }^{19,20}$. Es posible afirmar que el PPG pudo haber facilitado el acceso de las gestantes más necesitadas del servicio, mejorando la recepción, atención y la calidad de la atención prenatal.

El estudio de las causas del fracaso en la prevención de la sífilis congénita refuerza la importancia de la atención prenatal en su reducción. Por lo tanto, es necesario que las mujeres gestantes sean captadas tempranamente por los servicios de salud, sometiéndose a todos los exámenes recomendados $\mathrm{y}$, si están infectadas, sean rápida y adecuadamente tratadas, así como sus parejas. Actualmente, a pesar de no implicar el uso de tecnología compleja o de grandes inversiones, sigue siendo un objetivo lograr erradicar o incluso reducir significativamente la presencia de la sífilis en la población ${ }^{17}$.

Experiencias en diversas partes del mundo muestran que la manera más efectiva, sencilla y económica de prevenir la sífilis es informar a las mujeres gestantes sobre los riesgos de infección y guiarlas para que adquieran comportamientos preventivos ${ }^{13}$. El riesgo de sífilis puede ser eliminado, o por lo menos reducido significativamente, si las mujeres embarazadas siguen reglas simples de cuidado con el contagio directo, además de la higiene personal.

Se considera que en los protocolos de atención prenatal las gestantes también deben ser informadas de que toda medida preventiva reduce, pero no elimina completamente el riesgo de infección durante el embarazo. La prevención secundaria mediante exámenes trimestrales debe ser recomendada, especialmente a poblaciones de regiones con mayor prevalencia de la enfermedad ${ }^{18}$.

Adicionalmente, la importancia del número de registros realizados aporta información importante en lo que refiere a implementar medidas de prevención 219 de enfermedades de transmisión materno-fetal de alta prevalencia en nuestro contexto9. En este sentido, la primera consulta debe ocurrir tan pronto como sea posible, así como el número de consultas mínimas consideradas por el Ministerio de Salud (6) ${ }^{11}$.

\section{CONCLUSIONES}

Las tasas de prevalencia e incidencia de sífilis congénita fueron superiores a las reportadas por la OMS, las cuales están en torno de 1 caso por cada 1.000 nacidos vivos ${ }^{21}$.

El Programa de Protección a la Gestante contribuyó a verificar que la sífilis es un factor importante para el elevado número de morbilidad y mortalidad perinatal, como lo demuestra el número de casos de abortos, donde su frecuencia aumentó de $25,7 \%$ en las mujeres gestantes con cualquier tipo de alteración a un 35,3\% en las mujeres embarazadas solamente con sífilis, representando un aumento de 1,37 veces de los casos. El tamizaje durante el control prenatal, adecuadamente implementado, y a tiempo, podría disminuir y resolver en gran medida el problema de la sífilis congénita. Se puede decir que el PPG sirvió para observar el comportamiento epidemiológico de la sífilis y la cantidad de abortos en el Estado de Sergipe.

\section{AGRADECIMIENTOS}

Especialmente al grupo de evaluadores anónimos por la excelente contribución en la revisión y mejoras sugeridas al presente trabajo.

\section{CONFLICTO DE INTERÉS}

Los autores declaran que no tienen conflictos de interés relacionados con los contenidos de este artículo.

\section{REFERENCIAS}

1. Campos JEB. Significado laboratorial dos baixos títulos de VDRL (Venereal Disease Research Laboratories) para o diagnóstico da sífilis em gestantes, à luz das provas treponêmicas. Fundação Oswaldo Cruz. Instituto Fernandes Figueira; 2006.

2. Singh AE, Romanowski B. Syphilis: review with emphasis on clinical, epidemiologic, and some biologic features. Clin Microbiol Rev. 1999; 12(2): 187-209.

3. Walker DG, Walker GJ. Forgotten but not gone: the continuing scourge of congenital syphilis. Lancet Infect Dis. 2002; 2(7): 432-436.

4. Duarte G. Diagnóstico e conduta nas infecções 
ginecológicas e obstétricas. En: FUNPEC, editor. 2004: p. 147.

5. Hurtig AK, Nicoll A, Carne C, Lissauer T, Connor $\mathrm{N}$, Webster JP, et al. Syphilis in pregnant women and their children in the United Kingdom: results from national clinician reporting surveys 1994-7. BMJ. 1998; 317(7173): 1617-1619.

6. Wicher K, Horowitz HW, Wicher V. Laboratory methods of diagnosis of syphilis for the beginning of the third millennium. Microbes Infect. 1999; 1(12): 1035-1049.

7. Ronald A, Plourde P. Why are syphilis control programs failing? Int J Infect Dis. 1998; 2(3): 121122.

8. Leal Pasos MR. Doenças sexualmente transmissíveis. 4ed. Revinter. 1995.

9. Carellos EVM, Queiroz de Andrade GM, Pessoa de Aguiar RAL. Avaliação da aplicação do protocolo de triagem pré-natal para toxoplasmose em Belo Horizonte, Minas Gerais, Brasil: estudo transversal em puérperas de duas maternidades. Cad Saude Pública. 2008; 24(2): 391-401.

10. Lago EG, Ricard C, Harter K. Causas de falha na prevenção da sífilis congênita. Rev Med PUCRS. 2001; 11: 14-21.

11. Brasil. Ministério da Saúde. PNDST/AIDS-Estudo Sentinela Parturiente. 2004.

12. Brasil. Ministério da Saúde. Portaria $n^{\circ} .542$ de 22 de dezembro de 1986. Brasília: Diário Oficial da União, 24 dez.1986. Seção I.

13. Brasil. Ministério da Saúde. Secretaria de Políticas de Saúde. Coordenação Nacional de DST e AIDS. Projeto de eliminação da Sífilis Congênita. Manual de assistência e vigilância epidemiológica. Brasília, 1998.

14. Duarte G. Sífilis e gestação. En: Cunha SPC, Duarte G, Editores. Gestação de Alto Risco. 1 ${ }^{\mathrm{a} e d . ~ S a ̃ o ~ P a u l o: ~}$ Editora Médica e Científica, 1999. p. 277-88.

15. Araújo EC, Moura EFA, Ramos FLP, Holanda VGD. Sífilis congênita : incidência em recém-nascidos. J Pediatr. 1999; 75:119-125.

16. Lorenzi DRS, Madi JM, Pontalti L, Pölkin A, Ribas FE, Weissheimer L. Sífilis Congênita: revisão de 35 casos. GO Atual 2000; 9: 15-18.

17. Lago EG, Garcia PCR. Sífilis congênita: uma emergência emergente também no Brasil. J Pediatr. 2000; 76: 461-465.

18. Wendel GDJr, Sheffield JS, Hollier LM, Hill JB, Ramsey PS, Sánchez PJ. Treatment of syphilis in pregnancy and prevention of congenital syphilis. Clin Infect Dis. 2002; 35(Suppl 2): S200-209.

19. Siqueira de Carvalho D, Novaes HMD. Avaliação da implantação de programa de atenção pré-natal no Município de Curitiba, Paraná, Brasil: estudo em coorte de primigestas. Cad Saúde Pública. 2004; 20 : S220-230.

20. Coutinho T, Bustamante Teixeira MT, Dain S, Sayd JD, Coutinho LM. Adequação do processo de assistência pré-natal entre as usuárias do Sistema Único de Saúde em Juiz de Fora - MG. Rev Bras Ginecol Obstet. 2003; 25(10): 717-724.

21. Organização Mundial da Saúde. Classificação estatística internacional de doenças e problemas relacionados à saúde: 10a revisão (CID-10). Centro Colaborador da OMS para a Classificação de Doenças em Português/Edusp. 1ed.São Paulo, 1993. 